International Mathematical Forum, Vol. 9, 2014, no. 27, 1341 - 1346

HIKARI Ltd, www.m-hikari.com

http://dx.doi.org/10.12988/imf.2014.47130

\title{
The Order Theory in Limited BCI-Algebras
}

\author{
Xiaobo Cai and Yongchao Zhao
}

School of Information Science and Technology

Chuxiong Normal University, Chuxiong (675000), China

Copyright (c) 2014 Xiaobo Cai and Yongchao Zhao. This is an open access article distributed under the Creative Commons Attribution License, which permits unrestricted use, distribution, and reproduction in any medium, provided the original work is properly cited.

\begin{abstract}
In this paper, two kinds of equivalences in limited BCI- algebras will be found. One is from the partial ordering relation while the other one is from the $*-$ operation in BCI-algebras. And the internal relevance between the two kinds of equivalences will be discussed, too.
\end{abstract}

Keywords: limited BCI-algebra; order; equivalence

\section{1 the Basic Definitions}

BCI-algebra is a kind of logic algebras which is a new class of algebra systems and abstracted from the logic systems. The first recognized logic algebra is Boolean algebra. The BCI-algebra is found by japanese mathematician K.Iseki in 1960s.

Definition $1.1[1] A(2,0)$-type algebra $<X ; *, 0>$ would be called as a BCIalgebra, if it fulfils:

$B C I-1:((x * y) *(x * z)) *(z * y)=0$;

$B C I-2:(x *(x * y)) * y=0$;

$B C I-3: x * x=0$;

$B C I-4: x * y=y * x=0 \Leftrightarrow x=y$.

After its own birth, BCI-algebras have been studied very deeply by many researchers from different countries.

As the title, in this paper, only limited BCI-algebras will be studied. A limited BCI-algebra means in which one there are only limited elements. 
If we mark $x * y=0$ as $x \leq y$ in it, a BCI-algebra would be a partial ordering set. But this relevance is not a one to one correspondence. It's clearly that a partial ordering set could correspond to several BCI-algebras generally.

As that there would be some minimal elements in a partial ordering set, in BCI-algebras there must be atoms.

Definition 1.2 [1]Supposing $\langle X ; *, 0\rangle$ is a BCI-algebra, while $\alpha \in X$. $\alpha$ would be called as an atom of $X$ while $x * a=0 \Rightarrow x=a$. The set of all the atoms of $X$ is marked as $L(X)$.

It's clearly that the atoms of a BCI-algebra are exactly the minimal elements in $X$ as a partial ordering set.

And depends on the conception of atoms, the definition of branches has been found as follows.

Definition 1.3 [1]Supposing $\langle X ; *, 0\rangle$ is a BCI-algebra, while $\alpha$ is an atom of $X$. The set $\{x \in X \mid \alpha * x=0\}$ is named as a branch of $X$, marked as $l_{\alpha}$.

\section{Two Kinds of Equivalences}

\section{1 the One From the Partial Ordering Relation}

In the Fuzzy Set theory, there are two conceptions of cut set and strong cut set.[2] Analogously, we could define the cut sets and strong cut sets in BCIalgebras as follows.

Definition 2.1 Supposing $\langle X ; *, 0\rangle$ is a BCI-algebra, while $\alpha \in X$. The set $\{x \in X \mid x * \alpha=0\}$ is named as the $\alpha$-cut set of $X$, marked as $X_{\alpha}$.

Definition 2.2 Supposing $\langle X ; *, 0\rangle$ is a BCI-algebra, while $\alpha \in X$. The set $\{x \in X \mid x * \alpha=0, x \neq \alpha\}$ is named as the strong $\alpha$-cut set of $X$, marked as $X_{S \alpha}$.

Depends on these two definitions, some results are clearly as follows.

Theorem 2.1 Supposing $\langle X ; *, 0>$ is a BCI-algebra, $\alpha \in X, b \in L(X)$, then

$$
\alpha \in l_{b} \Leftrightarrow b \in X_{\alpha} .
$$
$X_{\alpha}$

Proof. $\alpha \in l_{a}=\{x \in X \mid b * x=0\} \Leftrightarrow b * \alpha=0 \Leftrightarrow b \in\{x \in X \mid x * \alpha=0\}=$

Theorem 2.2 Supposing $\langle X ; *, 0\rangle$ is a BCI-algebra, $\alpha, \beta \in X$, then

$$
X_{\alpha} \cap X_{\beta} \neq \emptyset \Leftrightarrow \exists m \in L(X) \quad \text { s.t. } \alpha, \beta \in l_{m}
$$


Proof." $\Rightarrow ":$ Supposing $r \in X_{\alpha} \cap X_{\beta}$ while $r \in l_{m}$. Because $r \in X_{\alpha} \cap$ $X_{\beta}, r * \alpha=0$ and $r * \beta=0$; Because $r \in l_{m}, m * r=0$. So, $m * \alpha=0$ and $m * \beta=0$, which means $\alpha \in l_{m}$ and $\beta \in l_{m}$.

$" \Leftarrow "$ : Because $\alpha \in l_{m}, m \in X_{\alpha}$; in the same argument, $m \in X_{\beta}$. As a result, $m \in X_{\alpha} \cap X_{\beta}$ which means $X_{\alpha} \cap X_{\beta} \neq \emptyset$.

Finally, in summary, we get the theorem.

Theorem 2.3 Supposing $\langle X ; *, 0>$ is a BCI-algebra, $\alpha, \beta \in X$, then:

$$
\alpha * \beta=0 \Leftrightarrow X_{\alpha} \subset X_{\beta}
$$

Proof.Supposing $x \in X_{\alpha}$, as $\alpha * \beta=0$, so $x * \beta \leq x * \alpha=0$, which means $x \in X_{\beta}$ for 0 is the least element in a BCI-algebra; as $X_{\alpha} \subset X_{\beta}$, so $\alpha \in X_{\alpha} \subset X_{\beta}$, which means $\alpha * \beta=0$.

But if change the cut set into strong cut set in it, this proposition will be not tenable.

Finally, according to the definitions of cut set and strong cut set in BCIalgebra, we could definite ordering number in BCI-algebra as follows.

Definition 2.3 Supposing $<X ; *, 0>$ is a limited BCI-algebra, $\alpha \in X$, $\lambda(\alpha)=\left|X_{\alpha}\right|$ is named as the ordering number of $\alpha$.

It's easy to notice that the ordering number of $\alpha$ is actually the number of the elements which are "smaller" than $\alpha$.

And we could definite the first kind of equivalence.

Definition 2.4 Supposing $\langle X ; *, 0\rangle$ is a limited BCI-algebra, $\alpha, \beta \in X$. If $\lambda(\alpha)=\lambda(\beta)$, it will be termed as that $\alpha$ and $\beta$ are $\lambda$-equivalent, marked as $\alpha \doteq \beta$.

It's too obviously to be proved that this relationship ". $"$ is indeed equivalence while it conform to the three conditions of equivalence.

\section{2 the Other One From the $*$-Operation}

As the it is the only operation in BCI-algebra, we want to find another new regularity of the $*$-operation. So we get the follows.

Definition 2.5 Supposing $\langle X ; *, 0\rangle$ is a limited BCI-algebra, $\alpha, \beta \in X$. If $\exists m, n \in L(X)$ s.t. $\alpha * m=\beta, \beta * n=\alpha$, it will be marked as $\alpha \approx \beta$.

And what's more.

Definition 2.6 Supposing $\langle X ; *, 0>$ is a limited BCI-algebra, $\alpha, \beta \in X$. It will be marked as $\alpha \sim \beta$, while $\exists x_{1}, x_{2}, \ldots, x_{n} \in X$ s.t. $\alpha \approx x_{1} \approx x_{2} \approx \cdots \approx$ $x_{n} \approx \beta$. 
It's easy to notice that the relation $\approx$ and $\sim$ in BCI-algebras are both equivalences. But as it's more universal, the relation $\sim$ will be our major subject.

According to the relation $\sim$ in BCI-algebras, we have found some results as the follows.

Theorem 2.4 Supposing $\langle X ; *, 0\rangle$ is a limited BCI-algebra, $\alpha, \beta \in X$ and $\alpha \sim \beta$. Then we have

$$
\alpha \in L(X) \Leftrightarrow \beta \in L(X) .
$$

Proof.Supposing $\alpha \in L(X)$, which means $\alpha$ is an atom. Because $\alpha \sim \beta$, $\exists x_{1}, x_{2}, \ldots, x_{n} \in X$ s.t. $\alpha \approx x_{1} \approx x_{2} \approx \cdots \approx x_{n} \approx \beta$. As $\alpha \approx x_{1}, \exists \mu \in L(X)$ s.t. $\alpha * \mu=x_{1}$. As

$$
0 *\left(0 * x_{1}\right)=0 *(0 *(\alpha * \mu))=\alpha * \mu=x_{1}
$$

$x_{1}$ is an atom which means $x_{1} \in L(X)$ [1].In the same argument, $\beta$ is an atom. And similarly, this theorem could be proved.

Theorem 2.5 Supposing $\left\langle X ; *, 0>\right.$ is a limited BCI-algebra, $\alpha \in l_{c} \subset$ $X, \beta \in l_{d} \subset X$. Then we have

$$
\alpha \sim \beta \Rightarrow c \sim d
$$

Proof.Because $\alpha \sim \beta, \exists x_{1}, x_{2}, \ldots, x_{n} \in X$ s.t. $\alpha \approx x_{1} \approx x_{2} \approx \cdots \approx x_{n} \approx$ $\beta$. As $\alpha \approx x_{1}, \exists \mu, \nu \in L(X)$ s.t. $\alpha * \mu=x_{1}, x_{1} * \nu=\alpha$. Supposing $x_{1} \in l_{e}$, then we have the follows

$$
\begin{aligned}
& c * \mu=(0 *(0 * \alpha)) *(0 *(0 * \mu))=0 *(0 *(\alpha * \mu))=0 *\left(0 * x_{1}\right)=e \\
& e * \nu=\left(0 *\left(0 * x_{1}\right)\right) *(0 *(0 * \nu))=0 *\left(0 *\left(x_{1} * \nu\right)\right)=0 *(0 * \alpha)=c
\end{aligned}
$$

which means $c \approx e$. Similarly, this theorem could be proved.

In these two theorems, replacing $\sim$ with $\approx$, they will be also right. They are the more particular forms.

More results will be given in the next section.

\section{3 the Internal Relevance between the Two Kinds of Equivalences and the Epilogue}

Depending on the two kinds of equivalences in BCI-algebras, we get some results as follows.

Theorem 3.1 Supposing $<X ; *, 0>$ is a limited BCI-algebra, $\alpha, \beta \in X$.

$$
\alpha \approx \beta \Rightarrow \lambda(\alpha)=\lambda(\beta) \Leftrightarrow \alpha \doteq \beta
$$


Proof.We just show the proof for the first form. Because $\alpha \approx \beta, \exists m, n \in$ $L(X)$ s.t. $\alpha * m=\beta, \beta * n=\alpha$.Assuming $x \in X_{\alpha}$ which means $x * \alpha=0$, then $(x * m) * \beta \leq(\alpha * m) * \beta=\beta * \beta=0$ which means $x * m \in X_{\beta}$. And as $m \in L(X), x \neq y \Rightarrow x * m \neq y * m$. So, $\lambda(\alpha) \leq \lambda(\beta)$. In the same argument, $\lambda(\beta) \leq \lambda(\alpha)$. As a result, $\lambda(\alpha)=\lambda(\beta)$ which means $\alpha \doteq \beta$.

And what's more.

Theorem 3.2 Supposing $\langle X ; *, 0\rangle$ is a limited BCI-algebra, $\alpha, \beta \in X$.

$$
\alpha \sim \beta \Rightarrow \lambda(\alpha)=\lambda(\beta) \Leftrightarrow \alpha \doteq \beta
$$

Proof.Obviously.

These should be the most important result in our studying. But its inverse proposition is not right. Instead of it, we have a theorem as the follow.

Theorem 3.3 Supposing $<X ; *, 0>$ is a limited BCI-algebra in which $x * z=$ $y * z \neq 0 \Rightarrow x=y, \alpha \in l_{m}, \beta \in l_{n}$.

$$
\left.\begin{array}{r}
\alpha \doteq \beta ; \\
\left\{x \in l_{m} \mid \lambda_{x}=\lambda_{\alpha}\right\}=\{\alpha\} ; \\
\left\{x \in l_{n} \mid \lambda_{x}=\lambda_{\beta}\right\}=\{\beta\} ; \\
m \approx n
\end{array}\right\} \Rightarrow \alpha \approx \beta
$$

Proof.Supposing $u, v \in L(X)$ and $m * u=n, n * v=m$. Let $X_{\alpha} * u=$ $\left\{x * u \mid x \in X_{\alpha}\right\}$, then $\left|X_{\alpha} * u\right|=\left|X_{\text {alpha }}\right|$. So $\lambda(\alpha * u)=\lambda(\alpha)=\lambda(\beta)$. And as $n *(\alpha * u)=(m * u) *(\alpha * u) \leq m * \alpha=0$, we have $\alpha * u \in l_{n}$. So $\alpha * u=\beta$. In the same argument, $\beta * v=\alpha$. In summary, $\alpha \approx \beta$.

And as a special case, we have the follow.

Theorem 3.4 Supposing $\langle X ; *, 0\rangle$ is a limited BCI-algebra.

$$
m, n E(X) \Rightarrow m \approx n
$$

Proof.Let $u=m * n$ and $v=n * m$, then $m * u=m *(m * n)$ and $n * v=n *(n * m)$. Because $(m * u) * n=(m *(m * n)) * n=0$, we have $m * u \leq n$. As $n$ is an atom, $m * u=n$. In the same argument, $n * v=m$. Finally, in summary, $m \approx n$.

Theorem 3.5 Supposing $\langle X ; *, 0>$ is a limited BCI-algebra in which $x * z=$ $y * z \neq 0 \Rightarrow x=y$ and

$$
\alpha \neq \beta, \lambda(\alpha)=\lambda(\beta) \Rightarrow X_{\alpha} \cap X_{\beta}=\emptyset .
$$

Then, as a result, we have:

$$
\alpha \approx \beta \Leftrightarrow \alpha \doteq \beta
$$

Proof.Obviously with the last theorems. 


\section{References}

[1] Jie Meng, Yonglin Liu. BCI-algebra Introduction, Shaanxi Science and Technology Press,2001.

[2] L.A. Zadeh, Fuzzy sets, Information and Control,1965.8:338-353.

Received: July 11, 2014 